\title{
Microwave-assisted synthesis and evaluation of antibacterial activity of novel 6-fluoroaryl-[1,2,4]triazolo[1,5-a]pyrimidines
}

\author{
Egor V. Verbitskiy, ${ }^{a, b, *}$ Svetlana A. Baskakova, ${ }^{a}$ Nikolay A. Rasputin, ${ }^{a}$ \\ Natal'ya A. Gerasimova, ${ }^{c}$ Polina G. Amineva, ${ }^{c}$ Natal'ya P. Evstigneeva ${ }^{c}$, \\ Natal'ya V. Zil'berberg, ${ }^{c}$ Nikolay V. Kungurov, ${ }^{c}$ Marionella A. Kravchenko, ${ }^{d}$ \\ Sergey N. Skornyakov, ${ }^{d}$ Gennady L. Rusinov ${ }^{a, b}$ Oleg N. Chupakhin, ${ }^{a, b}$ \\ and Valery N. Charushin ${ }^{a, b}$ \\ a Postovsky Institute of Organic Synthesis, Ural Branch of the Russian Academy of Sciences, \\ S. Kovalevskoy Str. 22, Ekaterinburg, 620990, Russia \\ ${ }^{b}$ Ural Federal University, Mira St. 19, Ekaterinburg, 620002, Russia \\ ${ }^{c}$ Ural Research Institute for Dermatology, Venereology and Immunopathology, \\ Scherbakova St. 8, Ekaterinburg, 620076, Russia \\ ${ }^{d}$ Ural Research Institute for Phthisiopulmonology, 22 Parts'ezda St. 50, \\ Ekaterinburg, 620039, Russia \\ E-mail: Verbitsky@ios.uran.ru
}

DOI: http://dx.doi.org/10.3998/ark.5550190.p009.731

\begin{abstract}
A series of 6-fluoroaryl substituted $[1,2,4]$ triazolo[1,5- $a]$ pyrimidines have been synthesized by using the microwave-assisted Suzuki cross-coupling reaction from readily available 6-bromo$[1,2,4]$ triazolo[1,5- $a]$ pyrimidine. The antimicrobial activity of new compounds has been evaluated in vitro against Mycobacterium tuberculosis $\mathrm{H}_{37} \mathrm{Rv}$ and gram-negative (Neisseria gonorrhoeae ATCC 49226) bacteria.
\end{abstract}

Keywords: $[1,2,4]$ triazolo[1,5-a]pyrimidines, Suzuki cross-coupling, microwave irradiation, antimycobacterial activity, anti-gonorrhea activity

\section{Introduction}

The need for the development of new antibacterial agents can hardly be overestimated. Such global scale phenomena as the emergence of multi-drug-resistance (MDR), and extensive drug-resistance (XDR), have raised the severe concern of the world healthcare community in the last few decades. The US Center for Disease Control and Prevention (CDC) has classified "high priority antibioticresistant bacteria" that include Mycobacterium tuberculosis (MDR, XDR), Neisseria gonorrhoeae, 
Staphylococcus aureus (MRSA), Clostridium difficile, Streptococcus pneumoniae, Klebsiella species, Acinetobacter, Campylobacter and Salmonella. ${ }^{1}$ This increased resistance demands the discovery and development of novel antimicrobial leads with high efficacy. ${ }^{2}$

Azoloazines belong to one of the most important groups of nitrogen-containing condensed heterocyclic systems. ${ }^{3}$ Being analogues of DNA purine bases, they can be regarded as plausible substrates for enzymatic biochemical processes. ${ }^{4}$ In particular, derivatives of $[1,2,4]$ triazolo$[4,3-a]$ pyrimidines have recently been reported as potential antibacterials. ${ }^{5-8}$ On the other hand, there are a few publications dealing with biological activities of $[1,2,4]$ triazolo[1,5- $a]$-pyrimidines as anxiolytic ${ }^{9}$ and antileukemia agents. ${ }^{10,11}$ Although 6-bromo-[1,2,4]triazolo[1,5-a]pyrimidine was first synthesized in 1961 by Makisumi, ${ }^{12}$ no publications concerning the reactivity of this compound in the Suzuki cross-coupling have so far been reported in the literature.

A typical procedure for preparation of 6-substituted $[1,2,4] \operatorname{triazolo}[1,5-a]$ pyrimidines $(3)$ is by condensation of $2 H$-[1,2,4]triazol-3-amine (1) with a 2-substituted malonaldehyde (2) or its synthetic equivalent (Scheme 1). ${ }^{9,13,14}$

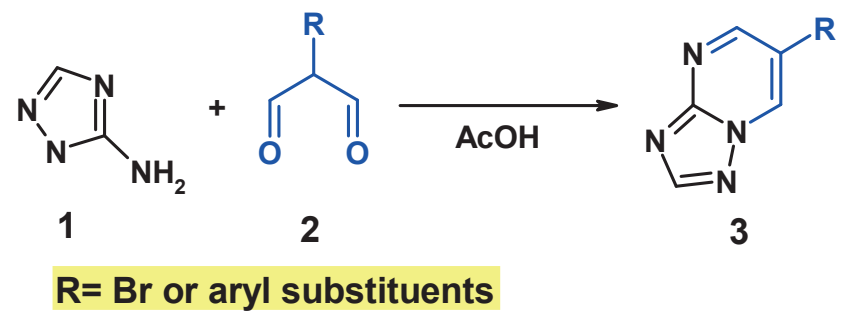

Scheme 1. Synthesis of 6-substituted $[1,2,4]$ triazolo[ $1,5-a]$ pyrimidines (3) by condensation of $2 H$-[1,2,4]triazol-3-amine (1) with 2-substituted malondialdehydes (2)

It is known that fluorine-containing heterocycles, especially fluoroquinolones, have gained attention as effective antibacterial agents. ${ }^{15-19} \mathrm{We}$ have recently elucidated how a fluorine atom (or $\mathrm{CF}_{3}$-group), incorporating at various positions a 5-(fluoroaryl) substituent in 4-(hetero)arylpyrimidines can affect their antibacterial activity against Mycobacterium tuberculosis and other pathogenic strains such as $M$. avium and M. terrae. ${ }^{20}$

In this communication we report the synthesis of novel 6-fluoroaryl substituted $[1,2,4]$ triazolo[1,5-a]pyrimidines using microwave-assisted Suzuki cross-coupling, and present data on their antimicrobial activities in vitro against Mycobacterium tuberculosis $\mathrm{H}_{37} \mathrm{Rv}$ and the gram-negative Neisseria gonorrhoeae ATCC 49226 bacteria. 


\section{Results and Discussion}

It has been shown that 5-bromopyrimidines react smoothly with a number of (hetero)arylboronic and trans-2-styrylboronic acids under microwave irradiation conditions to give the corresponding Suzuki cross-coupling products in high yields..$^{20-25}$

We have exploited this protocol for the synthesis of 6-phenyl- (5a) and 6-(fluoroaryl)$[1,2,4]$ triazolo[1,5-a]pyrimidines (5b-j). Indeed, compounds 5a-f were obtained in good yields by reacting the readily-available 6-bromo-[1,2,4]triazolo[1,5-a]pyrimidine (3) with phenylboronic (4a) and various fluorinated phenylboronic acids (4b-j) under microwave irradiation, using 1,4dioxane- $\mathrm{H}_{2} \mathrm{O}(4: 3)$ as solvent, and $\mathrm{K}_{2} \mathrm{CO}_{3}$ and $\mathrm{Pd}\left(\mathrm{PPh}_{3}\right)_{4}$, as catalyst (Scheme 2, Table 1).

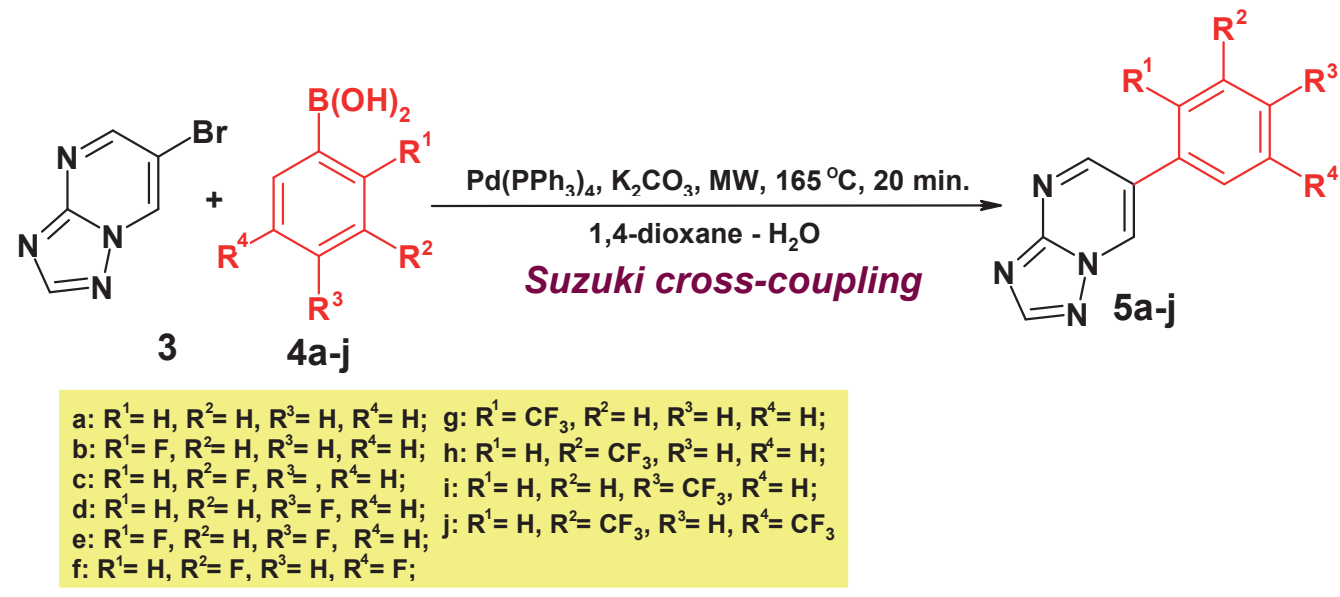

Scheme 2. Synthesis of 6-phenyl- and 6-(fluorophenyl)-[1,2,4]triazolo[1,5-a]pyrimidines (5a-j)

Table 1. Characteristics of 6-aryl-[1,2,4]triazolo[1,5-a]pyrimidines (5a-j)

\begin{tabular}{ccccc}
\hline Entry & Compds & 6-Substituent & $\begin{array}{c}\text { Isolated } \\
\text { Yield } \\
\mathbf{( \% )}\end{array}$ & Mp $\left({ }^{\circ} \mathbf{C}\right)$ \\
\hline 1 & $\mathbf{5 a}$ & phenyl & 75 & $166-168$ \\
2 & $\mathbf{5 b}$ & 2-fluorophenyl & 57 & 157 \\
3 & $\mathbf{5 c}$ & 3-fluorophenyl & 67 & $200-202$ \\
4 & $\mathbf{5 d}$ & 4-fluorophenyl & 70 & 234 \\
5 & $\mathbf{5 e}$ & 2,4-difluorophenyl & 73 & $187-188$ \\
6 & $\mathbf{5 f}$ & 3,5-difluorophenyl & 68 & $188-190$ \\
7 & $\mathbf{5 g}$ & 2-(trifluoromethyl)phenyl & 68 & $125-126$ \\
8 & $\mathbf{5 h}$ & 3-(trifluoromethyl)phenyl & 65 & 175 \\
9 & $\mathbf{5 i}$ & 4-(trifluoromethyl)phenyl & 60 & $209-210$ \\
10 & $\mathbf{5 j}$ & 3,5-bis(trifluoromethyl)phenyl & 74 & $166-168$ \\
\hline
\end{tabular}


Unequivocal evidence for the structure of compounds $\mathbf{5 a - j}$ was obtained by means of ${ }^{1} \mathrm{H}$ and ${ }^{13} \mathrm{C}$ NMR spectroscopy, as well as by X-ray crystallographic analysis carried out on 6-[3-(trifluoromethyl)phenyl][1,2,4]triazolo[1,5-a]pyrimidine (5h) (Fig. 1).

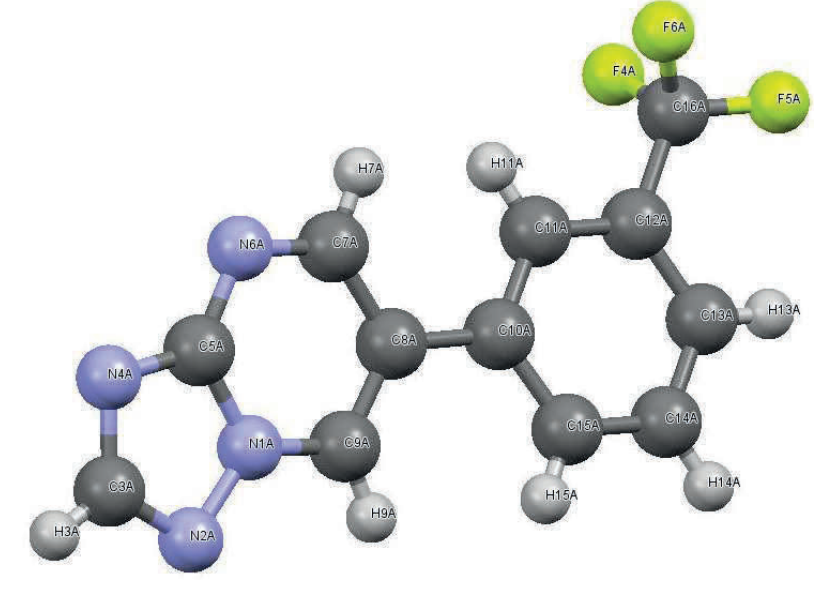

Figure 1. Mercury ${ }^{26}$ representation of the X-ray crystal structure of $\mathbf{5 h}$ with thermal ellipsoids of $50 \%$ probability.

Table 2. In vitro antibacterial activity of 6-substituted $[1,2,4]$ triazolo[1,5-a]pyrimidines (3 and 5a-j)

\begin{tabular}{cccc}
\hline & & \multicolumn{2}{c}{ Antibacterial activity (MIC), $\boldsymbol{\mu g} / \mathbf{m L}$} \\
\cline { 3 - 4 } Compds. & $\begin{array}{c}\text { Molecular } \\
\text { Mass }\end{array}$ & $\begin{array}{c}\text { Mycobacterium } \\
\text { tuberculosis } \mathbf{H}_{37} \mathbf{R v}\end{array}$ & $\begin{array}{c}\text { Neisseria } \\
\text { gonorrhoeae ATCC } \\
\text { (99226 }\end{array}$ \\
\hline $\mathbf{3}$ & 199.01 & 3.1 & $>1000$ \\
$\mathbf{5 a}$ & 196.21 & 3.1 & 1000 \\
$\mathbf{5 b}$ & 214.20 & 12.5 & 500 \\
$\mathbf{5 c}$ & 214.20 & 6.2 & 1000 \\
$\mathbf{5 d}$ & 214.20 & 12.5 & 1000 \\
$\mathbf{5 e}$ & 232.19 & 12.5 & 500 \\
$\mathbf{5 f}$ & 232.19 & 12.5 & 500 \\
$\mathbf{5 g}$ & 264.21 & 12.5 & 500 \\
$\mathbf{5 h}$ & 264.21 & 12.5 & 500 \\
$\mathbf{5 i}$ & 264.21 & 12.5 & 500 \\
$\mathbf{5 j}$ & 332.21 & 1.5 & 1000 \\
Isoniazid & 137.14 & 0.1 & - \\
Pyrazinamide & 123.12 & 12.5 & 0.015 \\
Ceftriaxone & 554.58 & - & 0.5 \\
Azithromycin & 748.98 & - & 32 \\
Spectinomycin & 332.35 & - & \\
\hline
\end{tabular}


Azolopyrimidines $\mathbf{3}$ and $\mathbf{5 a - j}$ were screened for their activity in vitro against Mycobacterium tuberculosis $\mathrm{H}_{37} \mathrm{Rv}$ and gram-negative (Neisseria gonorrhoeae ATCC 49226) bacteria, and the data on the minimal inhibitory concentrations (MICs) of these compounds are summarized in Table 2. Clinical drugs Isoniazid, Pyrazinamide and Azithromycin were taken as the reference compounds.

The antibacterial assay data show that the investigated $[1,2,4]$ triazolo $[1,5-a]$ pyrimidines showed moderate activity against Mycobacterium tuberculosis $\mathrm{H}_{37} \mathrm{Rv}$ in vitro. Displacement of the bromo atom of the pyrimidine ring with a fluoroaryl substituent leads to a reduction in antimycobacterial activity. On the other hand, variation of the fluoroaryl moiety in azolopyrimidines 5 has a negligible effect on their anti-gonorrheal activity, and the unexciting values of MIC, not exceeding $500 \mu \mathrm{g} / \mathrm{mL}$, have been obtained for compounds $\mathbf{5 b}, \mathbf{e}-\mathbf{i}$. Unfortunately, the poor results of antibacterial screening do not allow any conclusions to be drawn on structure-activity relationships.

\section{Conclusions}

We have demonstrated a convenient method for the synthesis of new 6-aryl substituted $[1,2,4]$ triazolo[1,5-a]pyrimidines, including fluorinated derivatives. Antibacterial studies have shown that fluoroaryl substituted derivatives are poorly active against Mycobacterium tuberculosis $\mathrm{H}_{37} \mathrm{Rv}$ and gram-negative (Neisseria gonorrhoeae ATCC 49226) bacteria, while 6-bromo- and 6phenyl-substituted $[1,2,4]$ triazolo[1,5-a]pyrimidines have a higher bacteriostatic effect, comparable with the first-line antituberculosis drug (Pyrazinamide).

Being not active enough to be therapeutics, compounds of this family can, nevertheless, be regarded as appropriate for further studies, aimed at development of effective agents to combat resistant forms of tuberculosis.

\section{Experimental Section}

General Information. All reagents and solvents were obtained from commercial sources and dried by using the standard procedures before use. 6-Bromo-[1,2,4]triazolo[ $1,5-a]$ pyrimidine was synthesized as described previously. ${ }^{12}$ Solvents $\left(1,4\right.$-dioxane and $\left.\mathrm{H}_{2} \mathrm{O}\right)$ for the microwave-assisted Suzuki cross-coupling reaction were deoxygenated by bubbling argon for $1 \mathrm{~h}$.

The ${ }^{1} \mathrm{H},{ }^{19} \mathrm{~F}$, and ${ }^{13} \mathrm{C}$ NMR spectra were recorded on a Bruker DRX-400 and Avance-500 instruments, using $\mathrm{Me}_{4} \mathrm{Si}$ and $\mathrm{C}_{6} \mathrm{~F}_{6}$ as internal standards. Elemental analyses were carried on a Eurovector EA 3000 automated analyzer. Melting points were determined on Boetius combined heating stages and were not corrected. 
The GC-MS analyses of all samples were carried out using an Agilent GC 7890A MS 5975C Inert $\mathrm{XL}$ EI/CI GC-MS spectrometer with a quadrupole mass-spectrometric detector with electron ionization $(70 \mathrm{eV})$.

Column chromatography was carried out using Alfa Aesar silica gel 0.040-0.063 mm (230-400 mesh). The progress of reactions and the purity of compounds were checked by TLC on Sorbfil plates (Russia), in which the spots were visualized with UV light ( $\lambda 254$ or $365 \mathrm{~nm}$ ).

Microwave experiments were carried out in a Discover unimodal microwave system (CEM, USA) with a working frequency of $2.45 \mathrm{GHz}$ and the microwave radiation power ranged from 0 to 300 $\mathrm{W}$. The reactions were carried out in a $10 \mathrm{~mL}$ reaction tube with the hermetic Teflon cork. The reaction temperature was monitored, using an inserted IR sensor by the external surface of the reaction vessel.

A suitable crystal of $\mathbf{5 h}$ was selected and XRD analysis was performed on a Xcalibur diffractometer using standard procedure $\left(\mathrm{MoK}_{\alpha}\right.$ graphite-monochromated irradiation, $\omega$-scanning with $1^{\mathrm{o}}$ steps). Compound $\mathbf{5} \mathbf{h}$ was solved and refined by using Olex2 program. ${ }^{27}$ Non-hydrogen atoms were refined in anisotropic approximation; $\mathrm{H}$-atoms were refined in isotropic approximation in riding model. The X-ray crystallography data for structure $\mathbf{5 h}$ reported in this paper have been deposited with Cambridge Crystallography Data Centre as supplementary publications CCDC no. 1483333 for $\mathbf{5 h}$. These data can be obtained free of charge from the Cambridge Crystallographic Data Centre via $w w w . c c d c . c a m . a c . u k / d a t a$ request/cif.

General procedure for the synthesis of 6 -aryl-[1,2,4]triazolo $[1,5-a]$ pyrimidine derivatives (5a-j). A solution of $\mathrm{K}_{2} \mathrm{CO}_{3}(346 \mathrm{mg}, 2.5 \mathrm{mmol})$ in $\mathrm{H}_{2} \mathrm{O}(3 \mathrm{~mL})$ was added to a mixture of a 6-bromo-[1,2,4]triazolo[1,5-a]pyrimidine (3) $(201 \mathrm{mg}, 1.0 \mathrm{mmol})$, arylboronic (4a-j) acid (1.2 $\mathrm{mmol})$ and $\mathrm{Pd}\left(\mathrm{PPh}_{3}\right)_{4}(58 \mathrm{mg}, 5 \mathrm{~mol} \%)$ in 1,4-dioxane $(4 \mathrm{~mL})$. The resulting mixture was irradiated in a microwave apparatus at $165^{\circ} \mathrm{C}(250 \mathrm{~W})$ for $20 \mathrm{~min}$. After that solvent was distilled off in vacuo, and the residue was purified by flash column chromatography (hexane/ethyl acetate, $1: 3)$ to afford the desired cross-coupling products $(\mathbf{5 a - j})$.

6-Phenyl-[1,2,4]triazolo[1,5-a]pyrimidine (5a). Yield 75\%, white solid, mp 166-168 ${ }^{\circ} \mathrm{C} .{ }^{1} \mathrm{H}$ NMR (500 MHz, DMSO- $\left.d_{6}\right): \delta=9.79(\mathrm{~d}, J=2.5 \mathrm{~Hz}, 1 \mathrm{H}), 9.29(\mathrm{~d}, J=2.5 \mathrm{~Hz}, 1 \mathrm{H}), 8.72(\mathrm{~s}, 1 \mathrm{H})$, 7.91-7.87 (m, 2H), 7.58-7.54 (m, 2H), $7.49(\mathrm{ddd}, J=7.4,3.8,1.2 \mathrm{~Hz}, 1 \mathrm{H}) \mathrm{ppm} .{ }^{13} \mathrm{C}$ NMR $(126$ MHz, DMSO- $\left.d_{6}\right): \delta=156.3,154.9,154.0,134.1,132.8,129.2,128.7,127.3,123.9 \mathrm{ppm} . \mathrm{GC} \mathrm{t}_{R}$ 22.39 min; MS m/z (rel intensity) $196\left(\mathrm{M}^{+}, 100\right)$. Anal. Calcd for $\mathrm{C}_{11} \mathrm{H}_{8} \mathrm{~N}_{4}(196.21)$ : C 67.34, H 4.11, N 28.55. Found: C 67.33, H 4.22, N 28.28.

6-(2-Fluorophenyl)-[1,2,4]triazolo[1,5-a]pyrimidine (5b). Yield $57 \%$, white solid, mp $157{ }^{\circ} \mathrm{C}$. ${ }^{1} \mathrm{H}$ NMR (500 MHz, DMSO-d $): \delta=9.70(\mathrm{~d}, J=1.7 \mathrm{~Hz}, 1 \mathrm{H}), 9.12(\mathrm{~d}, J=1.9 \mathrm{~Hz}, 1 \mathrm{H}), 8.76$ (s, $1 \mathrm{H}), 7.78(\mathrm{td}, J=7.9,1.5 \mathrm{~Hz}, 1 \mathrm{H}), 7.60-7.54(\mathrm{~m}, 1 \mathrm{H}), 7.47-7.38(\mathrm{~m}, 2 \mathrm{H}) \mathrm{ppm} .{ }^{13} \mathrm{C}$ NMR $(126$ MHz, DMSO- $\left.d_{6}\right): \delta=159.3\left(\mathrm{~d},{ }^{1} J_{\mathrm{C}, \mathrm{F}}=247.0 \mathrm{~Hz}\right), 156.5,155.8\left(\mathrm{~d},{ }^{3} J_{\mathrm{C}, \mathrm{F}}=3.9 \mathrm{~Hz}\right), 153.8,136.1(\mathrm{~d}$, $\left.{ }^{4} J_{\mathrm{C}, \mathrm{F}}=3.2 \mathrm{~Hz}\right), 131.2\left(\mathrm{~d},{ }^{4} J_{\mathrm{C}, \mathrm{F}}=2.7 \mathrm{~Hz}\right), 131.2(\mathrm{~s}), 125.3\left(\mathrm{~d},{ }^{4} J_{\mathrm{C}, \mathrm{F}}=3.5 \mathrm{~Hz}\right), 120.8\left(\mathrm{~d},{ }^{2} J_{\mathrm{C}, \mathrm{F}}=13.3\right.$ $\mathrm{Hz}), 118.9,116.2\left(\mathrm{~d},{ }^{2} J_{\mathrm{C}, \mathrm{F}}=21.7 \mathrm{~Hz}\right) \mathrm{ppm} .{ }^{19} \mathrm{~F}$ NMR $\left(470.5 \mathrm{MHz}, \mathrm{DMSO}-d_{6}\right): \delta=44.93-44.85$ (m, 1F). GC t $\mathrm{t}_{R} 22.09 \mathrm{~min}$; MS $\mathrm{m} / z$ (rel intensity) $214\left(\mathrm{M}^{+}, 100\right)$. Anal. Calcd for $\mathrm{C}_{11} \mathrm{H}_{7} \mathrm{FN}_{4}$ (214.20): C 61.68, H 3.29, N 26.16. Found: C 61.64, H 3.45, N 25.92. 
6-(3-Fluorophenyl)-[1,2,4]triazolo[1,5-a]pyrimidine (5c). Yield 67\%, white solid, mp 200-202 ${ }^{\circ} \mathrm{C} .{ }^{1} \mathrm{H}$ NMR $\left(500 \mathrm{MHz}, \mathrm{DMSO}-d_{6}\right): \delta=9.86(\mathrm{~d}, J=2.5 \mathrm{~Hz}, 1 \mathrm{H}), 9.32(\mathrm{~d}, J=2.5 \mathrm{~Hz}, 1 \mathrm{H}), 8.74$ $(\mathrm{s}, 1 \mathrm{H}), 7.84-7.80(\mathrm{~m}, 1 \mathrm{H}), 7.76(\mathrm{~d}, J=7.8 \mathrm{~Hz}, 1 \mathrm{H}), 7.60(\mathrm{td}, J=8.0,6.3 \mathrm{~Hz}, 1 \mathrm{H}), 7.33(\mathrm{td}, J=$ 8.3, $1.9 \mathrm{~Hz}, 1 \mathrm{H}) \mathrm{ppm} .{ }^{13} \mathrm{C}$ NMR $\left(126 \mathrm{MHz}, \mathrm{DMSO}-d_{6}\right): \delta=162.6\left(\mathrm{~d},{ }^{1} \mathrm{~J}_{\mathrm{C}, \mathrm{F}}=243.7 \mathrm{~Hz}\right), 156.5$, $154.8,154.1,135.15\left(\mathrm{~d},{ }^{3} J_{\mathrm{C}, \mathrm{F}}=8.4 \mathrm{~Hz}\right), 134.6,131.2\left(\mathrm{~d},{ }^{3} J_{\mathrm{C}, \mathrm{F}}=8.6 \mathrm{~Hz}\right), 123.3\left(\mathrm{~d},{ }^{4} J_{\mathrm{C}, \mathrm{F}}=2.7 \mathrm{~Hz}\right)$, $122.6\left(\mathrm{~d},{ }^{4} J_{\mathrm{C}, \mathrm{F}}=2.3 \mathrm{~Hz}\right), 115.5\left(\mathrm{~d},{ }^{2} J_{\mathrm{C}, \mathrm{F}}=21.1 \mathrm{~Hz}\right), 114.1\left(\mathrm{~d},{ }^{2} J_{\mathrm{C}, \mathrm{F}}=23.1 \mathrm{~Hz}\right) \mathrm{ppm} .{ }^{19} \mathrm{~F} \mathrm{NMR}$ (470.5 MHz, DMSO-d6): 50.47 (ddd, $1 \mathrm{~F}, J=10.5,8.9,6.2 \mathrm{~Hz}$ ). GC t $\mathrm{t}_{R} 22.22 \mathrm{~min}$; MS m/z (rel intensity) $214\left(\mathrm{M}^{+}, 100\right)$. Anal. Calcd for $\mathrm{C}_{11} \mathrm{H}_{7} \mathrm{FN}_{4}$ (214.20): C 61.68, H 3.29, N 26.16. Found: C 61.51, H 3.36, N 25.97.

6-(4-Fluorophenyl)-[1,2,4]triazolo[1,5-a]pyrimidine (5d). Yield 70\%, white solid, mp $234{ }^{\circ} \mathrm{C}$. ${ }^{1} \mathrm{H}$ NMR $\left(500 \mathrm{MHz}, \mathrm{DMSO}-d_{6}\right): \delta=9.78(\mathrm{~d}, J=2.5 \mathrm{~Hz}, 1 \mathrm{H}), 9.27(\mathrm{~d}, J=2.5 \mathrm{~Hz}, 1 \mathrm{H}), 8.72(\mathrm{~s}$, $1 \mathrm{H}), 8.03-7.86(\mathrm{~m}, 2 \mathrm{H}), 7.50-7.31(\mathrm{~m}, 2 \mathrm{H}) \mathrm{ppm} .{ }^{13} \mathrm{C}$ NMR $\left(126 \mathrm{MHz}, \mathrm{DMSO}-d_{6}\right): \delta=162.6(\mathrm{~d}$, $\left.{ }^{1} J_{\mathrm{C}, \mathrm{F}}=246.3 \mathrm{~Hz}\right), 156.3,154.8,153.9,134.1,129.5\left(\mathrm{~d},{ }^{3} J_{\mathrm{C}, \mathrm{F}}=8.4 \mathrm{~Hz}\right), 129.3\left(\mathrm{~d},{ }^{4} J_{\mathrm{C}, \mathrm{F}}=3.1 \mathrm{~Hz}\right)$, 123.0, $116.1\left(\mathrm{~d},{ }^{2} J_{\mathrm{C}, \mathrm{F}}=21.7 \mathrm{~Hz}\right) \mathrm{ppm} .{ }^{19} \mathrm{~F}$ NMR (470.5 MHz, DMSO- $\left.d_{6}\right): 49.19$ (tt, $1 \mathrm{~F}, J=8.9$, $5.4 \mathrm{~Hz}$ ). GC t $22.39 \mathrm{~min}$; MS m/z (rel intensity) $214\left(\mathrm{M}^{+}, 100\right)$. Anal. Calcd for $\mathrm{C}_{11} \mathrm{H}_{7} \mathrm{FN}_{4}$ (214.20): C 61.68, H 3.29, N 26.16. Found: C 61.54, H 2.67, N 25.91.

6-(2,4-Difluorophenyl)-[1,2,4]triazolo[1,5-a]pyrimidine (5e). Yield 73\%, white solid, mp 187$188{ }^{\circ} \mathrm{C} .{ }^{1} \mathrm{H}$ NMR $\left(500 \mathrm{MHz}, \mathrm{DMSO}-d_{6}\right): \delta=9.69(\mathrm{~d}, J=1.7 \mathrm{~Hz}, 1 \mathrm{H}), 9.09$ (t, $\left.J=2.0 \mathrm{~Hz}, 1 \mathrm{H}\right)$, $8.76(\mathrm{~s}, 1 \mathrm{H}), 7.84(\mathrm{td}, J=8.8,6.5 \mathrm{~Hz}, 1 \mathrm{H}), 7.58-7.47(\mathrm{~m}, 1 \mathrm{H}), 7.33(\mathrm{td}, J=8.5,1.8 \mathrm{~Hz}, 1 \mathrm{H}) \mathrm{ppm}$. ${ }^{13} \mathrm{C}$ NMR (126 MHz, DMSO- $\left.d_{6}\right): \delta=163.7\left(\mathrm{~d},{ }^{2} J_{\mathrm{C}, \mathrm{F}}=12.3 \mathrm{~Hz}\right), 161.7\left(\mathrm{~d},{ }^{2} J_{\mathrm{C}, \mathrm{F}}=12.3 \mathrm{~Hz}\right), 159.5$ $\left(\mathrm{dd},{ }^{1} J_{\mathrm{C}, \mathrm{F}}=249.9,{ }^{2} J_{\mathrm{C}, \mathrm{F}}=12.6 \mathrm{~Hz}\right), 156.5,155.7\left(\mathrm{~d},{ }^{4} J_{\mathrm{C}, \mathrm{F}}=3.7 \mathrm{~Hz}\right), 153.8,136.2\left(\mathrm{~d},{ }^{4} J_{\mathrm{C}, \mathrm{F}}=2.7\right.$ $\mathrm{Hz}), 132.6\left(\mathrm{dd},{ }^{3} J_{\mathrm{C}, \mathrm{F}}=10.0,{ }^{4} J_{\mathrm{C}, \mathrm{F}}=3.9 \mathrm{~Hz}\right), 117.5\left(\mathrm{dd},{ }^{3} J_{\mathrm{C}, \mathrm{F}}=13.7,{ }^{4} J_{\mathrm{C}, \mathrm{F}}=3.7 \mathrm{~Hz}\right), 112.5\left(\mathrm{dd},{ }^{2} J_{\mathrm{C}, \mathrm{F}}\right.$ $\left.=21.5,{ }^{4} J_{\mathrm{C}, \mathrm{F}}=3.7 \mathrm{~Hz}\right), 104.8\left(\mathrm{t},{ }^{2} J_{\mathrm{C}, \mathrm{F}}=26.3 \mathrm{~Hz}\right) \mathrm{ppm} .{ }^{19} \mathrm{~F}$ NMR $\left(470.5 \mathrm{MHz}, \mathrm{DMSO}-d_{6}\right): 54.45-$ $53.50(\mathrm{~m}, 1 \mathrm{~F}), 49.75(\mathrm{dd}, 1 \mathrm{~F}, J=18.8,9.1 \mathrm{~Hz})$. $\mathrm{GC} \mathrm{t}_{R} 21.72 \mathrm{~min}$; MS $m / z$ (rel intensity) $232\left(\mathrm{M}^{+}\right.$, 100). Anal. Calcd for $\mathrm{C}_{11} \mathrm{H}_{6} \mathrm{~F}_{2} \mathrm{~N}_{4}$ (232.19): C 56.90, H 2.60, N 24.13. Found: C 56.75, H 2.67, N 23.94 .

6-(3,5-Difluorophenyl)-[1,2,4]triazolo[1,5-a]pyrimidine (5f). Yield 68\%, white solid, mp 188$190{ }^{\circ} \mathrm{C} .{ }^{1} \mathrm{H}$ NMR $\left(500 \mathrm{MHz}, \mathrm{DMSO}-d_{6}\right): \delta=9.91(\mathrm{~d}, J=2.5 \mathrm{~Hz}, 1 \mathrm{H}), 9.35(\mathrm{~d}, J=2.5 \mathrm{~Hz}, 1 \mathrm{H})$, 8.76 (s, 1H), 7.84-7.68 (m, 2H), 7.38 (tt, $J=9.1,2.2 \mathrm{~Hz}, 1 \mathrm{H}) \mathrm{ppm} .{ }^{13} \mathrm{C}$ NMR (126 MHz, DMSO$\left.d_{6}\right): \delta=162.9\left(\mathrm{dd},{ }^{1} J_{\mathrm{C}, \mathrm{F}}=246.1,{ }^{3} J_{\mathrm{C}, \mathrm{F}}=13.7 \mathrm{~Hz}\right), 156.7,154.6,154.2,136.4\left(\mathrm{t},{ }^{2} J_{\mathrm{C}, \mathrm{F}}=10.6 \mathrm{~Hz}\right)$, 135.1, $121.5\left(\mathrm{t},{ }^{4} J_{\mathrm{C}, \mathrm{F}}=2.6 \mathrm{~Hz}\right), 110.5\left(\mathrm{dd},{ }^{2} J_{\mathrm{C}, \mathrm{F}}=20.4,{ }^{4} J_{\mathrm{C}, \mathrm{F}}=6.7 \mathrm{~Hz}\right), 104.0\left(\mathrm{t},{ }^{2} J_{\mathrm{C}, \mathrm{F}}=25.9 \mathrm{~Hz}\right)$ ppm. ${ }^{19} \mathrm{~F}$ NMR (470.5 MHz, DMSO-d $)$ ): 54.77-53.38 (m, 2F). GC t $21.65 \mathrm{~min}$; MS m/z (rel intensity) $232\left(\mathrm{M}^{+}, 100\right)$. Anal. Calcd for $\mathrm{C}_{11} \mathrm{H}_{6} \mathrm{~F}_{2} \mathrm{~N}_{4}$ (232.19): C 56.90, H 2.60, N 24.13. Found: C 56.76, H 2.56, N 23.97.

6-(2-Trifluoromethylphenyl)-[1,2,4]triazolo[1,5-a]pyrimidine (5g). Yield 68\%, white solid, mp 125-126 ${ }^{\circ} \mathrm{C} .{ }^{1} \mathrm{H}$ NMR $\left(500 \mathrm{MHz}, \mathrm{DMSO}-d_{6}\right): \delta=9.61(\mathrm{~d}, J=2.3 \mathrm{~Hz}, 1 \mathrm{H}), 8.88(\mathrm{~d}, J=1.8 \mathrm{~Hz}$, $1 \mathrm{H}), 8.77(\mathrm{~s}, 1 \mathrm{H}), 7.94(\mathrm{~d}, J=7.8 \mathrm{~Hz}, 1 \mathrm{H}), 7.83(\mathrm{t}, J=7.5 \mathrm{~Hz}, 1 \mathrm{H}), 7.75(\mathrm{t}, J=7.7 \mathrm{~Hz}, 1 \mathrm{H}), 7.67$ $(\mathrm{d}, J=7.6 \mathrm{~Hz}, 1 \mathrm{H}) \mathrm{ppm} .{ }^{13} \mathrm{C}$ NMR $\left(126 \mathrm{MHz}, \mathrm{DMSO}-d_{6}\right): \delta=156.6,154.8\left(\mathrm{~d},{ }^{1} J_{\mathrm{C}, \mathrm{F}}=223.4 \mathrm{~Hz}\right)$, 136.2, 133.4, 132.7, $131.9\left(\mathrm{~d},{ }^{4} J_{\mathrm{C}, \mathrm{F}}=1.6 \mathrm{~Hz}\right), 129.7,128.0\left(\mathrm{q},{ }^{2} J_{\mathrm{C}, \mathrm{F}}=29.4 \mathrm{~Hz}\right), 127.2,126.3(\mathrm{q}$, $\left.{ }^{4} J_{\mathrm{C}, \mathrm{F}}=5.2 \mathrm{~Hz}\right), 123.91\left(\mathrm{q},{ }^{1} J_{\mathrm{C}, \mathrm{F}}=274.0 \mathrm{~Hz}\right), 122.5 \mathrm{ppm} .{ }^{19} \mathrm{~F}$ NMR $\left(470.5 \mathrm{MHz}, \mathrm{DMSO}-d_{6}\right): 106.92$ 
(s, $\left.\mathrm{CF}_{3}\right) . \mathrm{GC} \mathrm{t}_{R} 21.27 \mathrm{~min}$; MS m/z (rel intensity) $264\left(\mathrm{M}^{+}, 100\right)$. Anal. Calcd for $\mathrm{C}_{12} \mathrm{H}_{7} \mathrm{~F}_{3} \mathrm{~N}_{4}$ (264.21): C 54.55, H 2.67, N 21.21. Found: C 54.61, H 2.80, N 21.05.

6-(3-Trifluoromethylphenyl)-[1,2,4]triazolo[1,5-a]pyrimidine (5h). Yield 65\%, white solid, mp $175^{\circ} \mathrm{C} .{ }^{1} \mathrm{H}$ NMR $\left(500 \mathrm{MHz}, \mathrm{DMSO}-d_{6}\right): \delta=9.94(\mathrm{~d}, J=2.5 \mathrm{~Hz}, 1 \mathrm{H}), 9.36(\mathrm{~d}, J=2.5 \mathrm{~Hz}, 1 \mathrm{H})$, $8.75(\mathrm{~s}, 1 \mathrm{H}), 8.29(\mathrm{~s}, 1 \mathrm{H}), 8.21(\mathrm{~d}, J=7.6 \mathrm{~Hz}, 1 \mathrm{H}), 7.85(\mathrm{~d}, J=7.8 \mathrm{~Hz}, 1 \mathrm{H}), 7.79$ (t, $J=7.7 \mathrm{~Hz}$, $1 \mathrm{H}) \mathrm{ppm} .{ }^{13} \mathrm{C}$ NMR $\left(126 \mathrm{MHz}, \mathrm{DMSO}-d_{6}\right): \delta=156.6,154.9,154.1,135.0,134.1,131.4,130.2$, $130.0\left(\mathrm{~d},{ }^{2} J_{\mathrm{C}, \mathrm{F}}=31.8 \mathrm{~Hz}\right), 125.2\left(\mathrm{dd},{ }^{3} J_{\mathrm{C}, \mathrm{F}}=7.1,{ }^{4} \mathrm{~J}_{\mathrm{C}, \mathrm{F}}=3.5 \mathrm{~Hz}\right), 124.1\left(\mathrm{q},{ }^{4} J_{\mathrm{C}, \mathrm{F}}=3.6 \mathrm{~Hz}\right), 124.05$ $\left(\mathrm{q},{ }^{1} J_{\mathrm{C}, \mathrm{F}}=272.7 \mathrm{~Hz}\right), 122.5 \mathrm{ppm} .{ }^{19} \mathrm{~F}$ NMR (470.5 MHz, DMSO-d $)$ : $101.62\left(\mathrm{~s}, \mathrm{CF}_{3}\right) . \mathrm{GC} \mathrm{t}_{R} 22.05$ min; MS $m / z$ (rel intensity) $264\left(\mathrm{M}^{+}, 100\right)$. Anal. Calcd for $\mathrm{C}_{12} \mathrm{H}_{7} \mathrm{~F}_{3} \mathrm{~N}_{4}$ (264.21): C 54.55, H 2.67, N 21.21. Found: C 54.53, H 2.56, N 21.04.

6-(4-Trifluoromethylphenyl)-[1,2,4]triazolo[1,5-a]pyrimidine (5i). Yield 60\%, white solid, mp 209-210 ${ }^{\circ} \mathrm{C} .{ }^{1} \mathrm{H}$ NMR $\left(500 \mathrm{MHz}, \mathrm{DMSO}-d_{6}\right): \delta=9.92(\mathrm{~d}, J=2.5 \mathrm{~Hz}, 1 \mathrm{H}), 9.34(\mathrm{~d}, J=2.5 \mathrm{~Hz}$, $1 \mathrm{H}), 8.76(\mathrm{~s}, 1 \mathrm{H}), 8.13(\mathrm{~d}, J=8.2 \mathrm{~Hz}, 2 \mathrm{H}), 7.92(\mathrm{~d}, J=8.3 \mathrm{~Hz}, 2 \mathrm{H}) \mathrm{ppm} .{ }^{13} \mathrm{C}$ NMR $(126 \mathrm{MHz}$, DMSO- $\left.d_{6}\right): \delta=155.7\left(\mathrm{~d},{ }^{1} J_{\mathrm{C}, \mathrm{F}}=228.8 \mathrm{~Hz}\right), 154.2,137.1,135.1,128.9\left(\mathrm{q},{ }^{2} J_{\mathrm{C}, \mathrm{F}}=32.1 \mathrm{~Hz}\right), 128.2$, $126.0\left(\mathrm{q},{ }^{4} J_{\mathrm{C}, \mathrm{F}}=3.7 \mathrm{~Hz}\right), 124.1$ (q, $\left.{ }^{1} J_{\mathrm{C}, \mathrm{F}}=272.1 \mathrm{~Hz}\right), 122.5 \mathrm{ppm} .{ }^{19} \mathrm{~F}$ NMR (470.5 MHz, DMSO$\left.d_{6}\right): 101.50\left(\mathrm{~s}, \mathrm{CF}_{3}\right) . \mathrm{GC} \mathrm{t}_{R} 22.35 \mathrm{~min} ; \mathrm{MS} \mathrm{m} / z$ (rel intensity) $264\left(\mathrm{M}^{+}, 100\right)$. Anal. Calcd for $\mathrm{C}_{12} \mathrm{H}_{7} \mathrm{~F}_{3} \mathrm{~N}_{4}$ (264.21): C 54.55, H 2.67, N 21.21. Found: C 54.41, H 2.61, N 21.08.

6-[3,5-Bis-(trifluoromethyl)phenyl]-[1,2,4]triazolo[1,5-a]pyrimidine (5j). Yield 74\%, white solid, mp 166-168 ${ }^{\circ} \mathrm{C} .{ }^{1} \mathrm{H}$ NMR $\left(500 \mathrm{MHz}, \mathrm{DMSO}-d_{6}\right): \delta=10.07(\mathrm{~d}, J=2.5 \mathrm{~Hz}, 1 \mathrm{H}), 9.45(\mathrm{~d}, J=$ $2.5 \mathrm{~Hz}, 1 \mathrm{H}), 8.78(\mathrm{~s}, 1 \mathrm{H}), 8.64(\mathrm{~s}, 2 \mathrm{H}), 8.22(\mathrm{~s}, 1 \mathrm{H}) \mathrm{ppm} .{ }^{13} \mathrm{C} \mathrm{NMR}\left(126 \mathrm{MHz}, \mathrm{DMSO}-d_{6}\right): \delta=$ $156.0\left(\mathrm{~d},{ }^{1} J_{\mathrm{C}, \mathrm{F}}=220.6 \mathrm{~Hz}\right), 154.2,135.9,135.8,131.1\left(\mathrm{q},{ }^{2} J_{\mathrm{C}, \mathrm{F}}=33.1 \mathrm{~Hz}\right), 128.4\left(\mathrm{~d},{ }^{4} J_{\mathrm{C}, \mathrm{F}}=2.8\right.$ $\mathrm{Hz}), 123.2\left(\mathrm{q},{ }^{1} J_{\mathrm{C}, \mathrm{F}}=273.1 \mathrm{~Hz}\right), 122.1\left(\mathrm{~d},{ }^{3} J_{\mathrm{C}, \mathrm{F}}=7.5 \mathrm{~Hz}\right), 122.1,121.2 \mathrm{ppm} .{ }^{19} \mathrm{~F}$ NMR $(470.5$ MHz, DMSO- $\left.d_{6}\right): 101.48$ (s, $2 \mathrm{CF}_{3}$ ). GC t $22.70 \mathrm{~min}$; MS: $\mathrm{m} / z$ (rel intensity) $332\left(\mathrm{M}^{+}, 100\right)$. Anal. Calcd for $\mathrm{C}_{13} \mathrm{H}_{6} \mathrm{~F}_{6} \mathrm{~N}_{4}$ (332.21): C 47.00, H 1.82, N 16.86. Found: C 47.13, H 1.98, N 16.71.

\section{Antimycobacterial assay.}

To evaluate the inhibitory efficiency of molecules on Mycobacterium tuberculosis (MTB), $M$. tuberculosis $H_{37} R v$, which is susceptible to all classical antituberculosis drugs, was used. The minimal inhibitory concentration (MIC) for M. tuberculosis $H_{37} R v$ for each compound was determined by a micro broth dilution method. All molecules tested were dissolved in dimethylsulfoxide and their $1 / 2$ dilutions were prepared in $5 \mathrm{~mL}$ tubes using Löwenstein-Jensen medium. A few colonies from freshly grown M. tuberculosis $H_{37} R v$ were suspended in Löwenstein-Jensen medium to obtain 1.0 McFarland turbidity and diluted ten times using the same medium and the tubes were incubated at $37^{\circ} \mathrm{C}$ medium with a different concentration of the tested molecule and to a positive control tube containing only clear growth medium. After 24 hours the tubes were placed in a vertical position and the free edge of the buried $0.3 \mathrm{~mL}$ of the substance in the test compounds concentrations: $12.5,6.2,3.1,1.5,0.7,0.37,0.15 \mu \mathrm{g} / \mathrm{mL}$. The tubes were then placed in an thermostat at a temperature of $37^{\circ} \mathrm{C}$ and incubated for 10 days. Growth estimate for the MTB were determined by standard methods, where the appearance of zones of growth retardation MTB (over $10 \mathrm{~mm}$ ) indicated the presence of tuberculostatic properties in concentration of the compounds under study. Penetration size stunting MTB (in $\mathrm{mm}$ ) is 
proportional to the degree of tuberculostatic activity. Growth delay of $100 \mathrm{~mm}$ or more is considered as a complete growth inhibition MTB. The multi-drug-resistant (MDR) tuberculosis strain have been isolated from tuberculosis patients in Ural Research Institute for Phthisiopulmonology (Russia). The minimal inhibitory concentrations against Mycobacterium avium, Mycobacterium terrae, and MDR tuberculosis strains were evaluated similarly.

\section{Anti-gonorrhea activity assay.}

The two fold serial dilution technique recommended by Clinical and Laboratory Standards Institute (CLSI) ${ }^{28}$ was used to evaluate the inhibitory efficiency of molecules on Neisseria gonorrhoeae. The medium for testing Neisseria gonorrhoeae consists of GC agar to which a 1\% defined growth supplement. Adjust the density of the suspension to contain $10^{8} \mathrm{CFU} / \mathrm{mL}$ by comparison with a 0.5 McFarland turbidity standard. For suspension using colonies from an overnight (20- to 24-hour) chocolate agar plate incubated in $5 \% \mathrm{CO}_{2} 36 \pm 1^{\circ}$. Dilute this suspension 1:10 in Muller-Hinton to give $10^{7} \mathrm{CFU} / \mathrm{mL}$. The test compounds concentrations: 1000, 500, 250, $125,62.5,31.2,15.0,7.5,3.8,1.9,0.99 \mu \mathrm{g} / \mathrm{mL}$ (solvent - DMSO, diluent $-\mathrm{H}_{2} \mathrm{O}$ and $\mathrm{GC}$ agar base).

\section{Acknowledgements}

The research was financially supported by the Russian Science Foundation (Project No. 15-1300077).

\section{References}

1. Chaudhary, A. S.; Jin, J.; Chen, W.; Tai, P. C.; Wang, B. Bioorg. Med. Chem. 2015, 23, 105. http://dx.doi.org/10.1016/j.bmc.2014.11.017

2. Rane, R. A.; Naphade, S. S.; Bangalore, P. K.; Palkar, M. B.; Shaikh, M. S.; Karpoormath, R. Bioorg. Med. Chem. Lett. 2014, 24, 3079 and references cited therein. http://dx.doi.org/10.1016/j.bmcl.2014.05.018

3. Li, J. J. Heterocyclic Chemistry in Drug Discovery; Wiley \& Sons, 2013; p. 720. ISBN: 978-1-118-14890-7.

4. Corey, E. J.; Kürti, L.; Czakó, B. Molecules and Medicine; Wiley \& Sons, 2008; p. 272. ISBN: 978-0-470-26096-8.

5. Prakash, O.; Bhardwaj, V.; Kumar, R.; Tyagi, P.; Aneja, K. R. Eur. J. Med. Chem. 2004, 39, 1073.

http://dx.doi.org/10.1016/j.ejmech.2004.06.011 
6. Kumar, R.; Nair, R. R.; Dhiman, S. S.; Sharma, J.; Prakash, O. Eur. J. Med. Chem. 2009, 44, 2260 . http://dx.doi.org/10.1016/j.ejmech.2008.06.004

7. Khera, M. K.; Cliffe, I. A.; Mathur, T.; Prakash, O. Bioorg. Med. Chem. Lett. 2011, 21, 2887. http://dx.doi.org/10.1016/j.bmcl.2011.03.075

8. Krishnaraj, T.; Muthusubramanian, S. J. Heterocyc. Chem. 2014, 51, E68. http://dx.doi.org/10.1002/jhet.1806

9. Dusza, J. P.; Hardy, R. A. Jr.; Albright, J. D. (1980) Patent US 4209621.

10. Li,Y.; Shen, M.; Zhang, Z.; Luo, J.; Pan, X.; Lu, X.; Long, H.; Wen, D.; Zhang, F.; Leng, F.; Li, Y.; Tu, Z.; Ren, X.; Ding, K. J. Med. Chem. 2012, 55, 10033. http://dx.doi.org/10.1021/jm301188x

11. Ren, X.; Pan, X.; Zhang, Z.; Wang, D.; Lu, X.; Li, Y.; Wen, D.; Long, H.; Luo, J.; Feng, Y.; Zhuang, X.; Zhang, F.; Liu, J.; Leng, F.; Lang, X.; Bai, Y.; She, M.; Tu, Z.; Pan, J.; Ding, K. J. Med. Chem. 2013, 56, 879. http://dx.doi.org/10.1021/jm301581y

12. Makisumi, Y. Chem. Pharm. Bull. 1961, 9, 808.

13. Petrich, S. A.; Qian, Z.; Santiago, L. M.; Gupton, J. T.; Sikorski, J. A. Heterocycles 1995, 40, 729. http://dx.doi.org/10.3987/COM-94-S61

14. Henrich, M.; Weil, T.; Hechenberger, M.; Müller, S.; Kauss, V.; Zembiro, R.; Erdmane, E. Smits, G. (2011) Patent WO 2011/015343 A1.

15. Nosova, E. V.; Mochulskaya, N. N.; Kotovskaya, S.K.; Lipunova, G. N.; Charushin, V.N. Heteroatom Chem. 2006, 17, 579.

http://dx.doi.org/10.1002/hc.20281

16. Zhou, Y.; Wang, J.; Gu, Z.; Wang, S.; Zhu, W.; Aceña, J. L.; Soloshonok, V.A.; Izawa, K.; Liu, H. Chem. Rev. 2016, 116, 422. http://dx.doi.org/10.1021/acs.chemrev.5b00392

17. V. N. Charushin, E. V., Nosova, G. N. Lipunova, O. N. Chupakhin, Fluoroquinolones: Synthesis and Application, Fizmatlit, Moscow, 2013.

18. V. N. Charushin, G. N. Lipunova, E. V. Nosova, O. N. Chupakhin, Fluoroquinolones: Synthesis and Application, in: V. Nenajdenko (Ed.), Fluorine in Heterocyclic Chemistry, Springer International Publishing, New York, 2014, p. 111-179.

19. Asif, M. Chemistry International 2015, 1, 134.

20. Verbitskiy, E. V.; Baskakova, S. A.; Kravchenko, M. A.; Skornyakov, S. N.; Rusinov, G. L.; Chupakhin, O. N.; Charushin, V. N. Bioorg. Med. Chem. 2016, in press. http://dx.doi.org/10.1016/j.bmc.2016.06.020

21. Verbitskiy, E. V.; Cheprakova, E. M.; Slepukhin, P. A.; Kodess, M. I.; Ezhikova, M. A.; Pervova, M. G.; Rusinov, G. L.; Chupakhin, O. N.; Charushin, V.N. Tetrahedron 2012, 68, 5445.

http://dx.doi.org/10.1016/j.tet.2012.04.095 
22. Verbitskiy, E. V.; Rusinov, G. L.; Charushin, V. N.; Chupakhin, O. N.; Cheprakova, E. M.; Slepukhin, P. A.; Pervova, M. G.; Ezhikova, M. A.; Kodess, M.I. Eur. J. Org. Chem. 2012, 6612.

http://dx.doi.org/10.1002/ejoc.201201035

23. Kravchenko, M. A.; Verbitskiy, E. V.; Medvinskiy, I. D.; Rusinov, G.L.; Charushin, V.N. Bioorg. Med. Chem. Lett. 2014, 24, 3118. http://dx.doi.org/10.1016/j.bmcl.2014.05.006

24. Cheprakova, E. M.; Verbitskiy, E. V.; Ezhikova, M. A.; Kodess, M. I.; Pervova, M. G.; Slepukhin, P. A.; Toporova, M. S.; Kravchenko, M. A.; Medvinskiy, I. D.; Rusinov, G. L.; Charushin, V. N. Russ. Chem. Bull. 2014, 63, 1350. http://dx.doi.org/10.1007/s11172-014-0602-y

25. Verbitskiy, E. V.; Cheprakova, E. M.; Slepukhin, P. A.; Kravchenko, M. A.; Skornyakov, S. N.; Rusinov, G. L.; Chupakhin, O.N.; Charushin, V.N. Eur. J. Med. Chem. 2015, 97, 225. http://dx.doi.org/10.1016/j.ejmech.2015.05.007

26. Mercury 3.3, available from http://www.ccdc.cam.ac.uk/mercury/.

27. Dolomanov, O. V.; Bourhis, L. J.; Gildea, R. J. ; Howard, J. A. K.; Puschmann, H. J. Appl. Cryst. 2009. 42, 339. DOI: $10.1107 / \mathrm{S} 0021889808042726$

28. Clinical and Laboratory Standards Institute. Performance Standards for Antimicrobial Susceptibility Testing; Twenty-Fourth Informational Supplement; Clinical and Laboratory Standards Institute: Wayne, PA, 2014. (http://www.cdc.gov/std/gonorrhea/arg/b88-feb2005.pdf). 